\title{
Discurso médico y estrategias de marketing de la industria farmacéutica en los procesos de medicación de la infancia en Argentina
}

\author{
Silvia Faraone \\ Alejandra Barcala² \\ Flavia Torricelli ${ }^{3}$ \\ Eugenia Bianchi ${ }^{4}$ \\ María Cecilia Tamburrino ${ }^{5}$
}

FARAONE, S. et al. Medical discourse and marketing strategies of the pharmaceutical industry in the process of medicalization of childhood in Argentina. Interface - Comunic., Saude, Educ., v.14, n.34, p.485-97, jul./set. 2010.

This paper explores at the processes of medicalization and medicinalization of childhood, using attention deficit disorder with or without hyperactivity $(\mathrm{ADD} / \mathrm{H})$ as the analyzer. The results from a qualitative study carried out by an interdisciplinary team during 2008 in four Argentine jurisdictions (Buenos Aires Metropolitan Area, Corrientes, Salta and Tierra del Fuego) are presented. Discourse from the field of medicine (pediatricians, childhoodadolescence psychiatrists and pediatric neurologists) regarding the diagnostic construct of $\mathrm{ADD} / \mathrm{H}$ and its therapeutic approach are explored, in relation to the public and private systems. In a complementary manner, the marketing mechanisms of pharmaceutical industry are investigated. Through the diagnostic construct of $A D D / H$, it can be seen how certain conducts and/or situations that had not been medicalized in the past have now become part of medical treatment, in which the main emphasis is on prescribing drugs as therapy.

Keywords: Childhood. Medicalization/ medicinalization. Psychoactive drugs.
Este artículo explora procesos de medicación incluso medicamentosa en la infancia, utilizando como analizador el Trastorno del Déficit de Atención con o sin Hiper-actividad (TDA/H). Se presentan los resultados de un estudio cualitativo realizado por un equipo interdisciplinario durante 2008 en cuatro jurisdicciones argentinas: Región Metropolitana de Buenos Aires, Corrientes, Salta y Tierra del Fueg. Se exploran los discursos del discursos campo médico - pediatras, psiquiatras infanto-juveniles y neurólogos infantiles - en torno de la construcción diagnóstica del TDH/A y su planteamiento terapéutica en los sistemas públicos y en el privado. De modo complementario se indaga acerca de los mecanismos de marketing de la industria farmacéutica. A través de la construcción de diagnóstico TDA/H es posible observar como determinadas conductas $\mathrm{y} / \mathrm{o}$ situaciones que antes no eran medicadas hoy forman parte del tratamiento médico, cuyo principal énfasis se encuentra en la prescripción de fármacos como terapéutica.

Palabras clave: Infancia. Medicación. Psicofármacos.
1 Instituto de Investigaciones Gino Germani, Facultad de Ciencias Sociales, Universidad de Buenos Aires. Uriburu 950, Ciudad Autónoma de Buenos Aires, Argentina. sfaraone@mail.fsoc.uba.ar

${ }^{2}$ Cátedra Salud Pública, Facultad de Psicología, Universidad de Buenos ${ }^{3}$ Cátedra Psicoanálisis: Psicología del Yo, Facultad de Psicología, Universidad de Buenos Aires. 4,5 Instituto de Investigaciones Gino Germani, Facultad de Ciencias Sociales, Universidad de Buenos Aires. 


\section{Introducción}

La medicalización es un objeto de estudio que viene siendo analizado desde hace varias décadas, en múltiples campos disciplinares y tradiciones de pensamiento: medicina, antropología, sociología, entre otros. La medicalización refiere al proceso progresivo mediante el cual el saber y la práctica médica incorporan, absorben y colonizan esferas, áreas y problemas de la vida social y colectiva que anteriormente estaban reguladas por otras instituciones, actividades o autoridades, como la familia, la religión, etc. (Foucault, 2001, 2000, 1996; Conrad, 1982; Illich, 1975). Medicalización, entonces, es el proceso histórico por el cual el campo médico se ocupa y trata diversos problemas - otrora no médicos - asociados a características intrínsecas de la vida; así, problemas de la vida son tratados en términos de problemas médicos como padecimientos, síndromes o enfermedades.

En este artículo nos interesa profundizar en una de las formas actuales que adopta la medicalización: la medicamentalización (Iriart, 2008); es decir, la medicación o el tratamiento farmacológico como respuesta frecuentemente exclusiva a ese proceso medicalizador. Para ello tomaremos un analizador particular: el Trastorno de Déficit de Atención con o sin Hiperactividad (TDA/H).

Entendemos por analizador una situación o un problema que al ser abordado hace visible una lógica de construcción social que trasciende esa situación o problema (Faraone, 2008). Pensar en el TDA/H como analizador nos lleva a considerar un fenómeno histórico más amplio, que actúa como horizonte de posibilidad para el accionar y alcance actual de la medicina.

Decimos que el TDA/H es un analizador privilegiado para conocer las características del proceso de medicalización hoy, por varias razones: en primer lugar, porque el blanco de las acciones es el niño, sujeto que históricamente ha estado en el centro de las estrategias de la medicalización (Foucault, $2006,2005,2000,1996)$; segundo, porque reformula un viejo problema, la conducta infantil, en términos acordes a las nuevas formas de gestión del disciplinamiento de las poblaciones, por eso, las técnicas que se despliegan pueden extenderse como metodología para el tratamiento de otras situaciones o problemas de adaptación a los modelos socialmente esperados y aceptados; y, por último, porque esta estrategia de medicalización incluye medicación en su tratamiento, lo cual abre una serie de consideraciones en relación al rol de las industrias farmacéuticas y la difusión de los fármacos como solución naturalizada.

Estas características permiten observar una serie de elementos que hacen del TDA/H un objeto singularmente relevante para el análisis de los procesos actuales de medicalización / medicamentalización en la infancia en la Argentina, debido a: por un lado, el aumento en este país de la importación de metilfenidato, según datos informados por la Administración Nacional de Medicamentos Alimentos y Tecnología Médica (ANMAT); por otro, la elaboración, por un grupo de expertos, de un documento en el año 2007 que alerta al Ministerio de Salud de la Nación acerca del importante número de niños diagnosticados con y tratados por este síndrome (Benasayag, 2007), del incremento de diagnósticos de niños cada vez más pequeños (Vasen, 2005), de la inespecificidad del diagnóstico (Benasayag, 2007; Janin, 2007), del tipo de medicación indicada - psicoestimulantes - y de las implicancias éticas del suministro de psicofármacos a tan corta edad (Brio, 2007); por último, la aparición, cada vez más frecuente, de publicaciones acerca de la dimensión de cronicidad de este padecimiento y/o síndrome en revistas tanto del campo médico, como del campo educativo y familiar (Scandar, 2007; Michanie, 2000b).

El presente trabajo está basado en los resultados de una investigación desarrollada durante el año 2008 a través de un convenio entre el Instituto de investigaciones Gino Germani de la Facultad de Ciencias Sociales de la Universidad de Buenos Aires y el Observatorio Argentino de Drogas de la Secretaría de Programación para la Prevención de la Drogadicción y la Lucha contra el Narcotráfico (SEDRONAR). Dicho estudio tuvo por objetivo analizar la problemática de consumo de psicotrópicos en niños diagnosticados con TDA/H y su relación con los procesos de medicalización/ medicamentalización. A través de un diseño cualitativo, se desarrolló el trabajo de campo en cuatro provincias argentinas, y se dividió el análisis en dos componentes: educación y salud. En el componente de educación, se analizaron las dinámicas, circuitos y actores involucrados en la detección, 
${ }^{6}$ Además de las autoras de este artículo participaron del proceso de investigación Isabel Ballesteros, médica pediatra, magister en epidemiología; Virginia López Casariego, médica pediatra y Constanza Leone, estudiante avanzada de la carrera de Ciencias de la Comunicación.

Para la organización de los puntos muestrales y selección de unidades de análisis se construyó un indicador utilizando dos variables: expendio de metilfenidato $y$ atomoxetina, $y$ población entre $0 \mathrm{y}$

14 años. Los datos de venta en farmacias fueron suministrados por la Confederación Farmacéutica Argentina (COFA). Debemos remarcar que este procedimiento fue sólo a los fines metodológicos y con el objeto de priorizar jurisdicciones para el trabajo de campo. No constituyó un dato válido ni de

consumo, ni de población

bajo tratamiento, sino sólo una construcción orientativa que nos permitió diseñar y priorizar los puntos nodales del trabajo de campo.

${ }^{8}$ Las dos primeras corresponden a provincias con "mayor expendio" de metilfenidato y atomoxetina por población de zero a 14 años y las siguientes integraban el listado de provincias que se encuentran dentro de

la categoría de "menor expendio" de dichas drogas para igual población. diagnóstico y abordaje del TDA/H dentro de la institución escolar; y en el componente de salud, se analizó la detección, diagnóstico, circuitos y abordajes dentro del campo médico.

En este artículo, trabajamos con algunos de los resultados correspondientes al campo médico. Este componente estuvo a cargo de un equipo interdisciplinario ${ }^{6}$, fue dirigido por la $\mathrm{Mg}$. Silvia Faraone y tuvo como sede el Instituto de Investigaciones Gino Germani.

\section{Objetivo general}

Analizar el proceso medicalización /medicamentalización en la infancia en Argentina a partir de un analizador: el TDA/H, su construcción diagnóstica y el abordaje basado en el suministro de psicofármacos, desde la perspectiva de los profesionales del campo médico.

Describir el reposicionamiento de la industria farmacéutica en los procesos de medicalización/medicamentalización a través del análisis de sus estrategias de marketing.

\section{Aspectos metodológicos}

Los resultados aquí expuestos corresponden a un estudio cualitativo donde se analiza el discurso de los agentes del campo médico en relación a los procesos de medicalización/medicalización de la infancia.

Para el estudio del campo médico se seleccionaron profesionales de la salud pediatras, psiquiatras infanto-juveniles y neurólogos infantiles - pertenecientes al subsector público y privado de salud. También se seleccionaron informantes claves pertenecientes a la industria farmacéutica - agentes de propaganda médica (APM) y líderes de opinión. La muestra fue intencional no representativa. Se realizaron un total de 45 entrevistas semi-estructuradas.

El trabajo de campo se realizó en cuatro provincias Argentinas?: Área Metropolitana (ciudad de Buenos Aires y primer Cordón del Conurbano bonaerense), Tierra del Fuego, Salta y Corrientes ${ }^{8}$.

\section{El TDA/H cono clasificación gnoseológica}

Según el Manual Diagnóstico y Estadístico de Salud Mental (DSM-IV, 1995), que tiene como objetivo proporcionar criterios diagnósticos específicos de los distintos trastornos mentales, el síndrome del TDA/H está incluido dentro de lo que denomina como "Trastornos por déficit de atención y comportamiento perturbador". El TDA/H puede presentarse en su tipo combinado (déficit de atención e hiperactividad), con predominio de déficit de atención, o con predominio hiperactivo-impulsivo.

El tratamiento farmacológico de este síndrome suele llevarse a cabo con metilfenidato, droga cuyo nombre comercial más conocido es la Ritalina ${ }^{\circledR}$. Se trata de un estimulante de acción similar a las anfetaminas, que por su potencialidad adictiva está incluido en el listado de psicofármacos de alta vigilancia controlados por la Junta Internacional de Fiscalización de Estupefacientes (JIFE) de la ONU. Su venta en el país requiere el uso de un recetario oficial que los médicos deben solicitar al Ministerio de Salud de la 
Nación. Las recetas se dan por triplicado y con archivo en la ANMAT. En los últimos años, el TDA/H también se trata con atomoxetina, droga que no requiere el mismo procedimiento que el metilfenidato, hecho que vuelve más difícil su control.

El TDA/H muestra dos grandes líneas de debate en la actualidad en la Argentina: por un lado, sobre el diagnóstico; y por otro, sobre el tratamiento. En lo que respecta al diagnóstico, lo que es puesto en discusión es la existencia o no del síndrome o trastorno. En relación al tratamiento, lo que se discute es el modo en que este síndrome o trastorno debe ser abordado, particularmente el uso de medicación como tratamiento de primera elección. Las posturas críticas al diagnóstico y al tratamiento con medicación afirman que es inadecuado - desde el punto de vista de la Salud Pública - unificar a todos los niños desatentos y/o inquietos en una clasificación psiquiátrica donde una categoría descriptiva pasa a ser explicativa de todo lo que le ocurre al niño.

\section{La medicalización/medicalización como construcción social}

La medicalización como proceso histórico es un tema muy amplio, que ha sido abordado desde múltiples campos y tradiciones de pensamiento. Acuñado por el filósofo, historiador y crítico social Ivan Illich, en su libro "Némesis médica: la expropiación de la salud" en el año 1975, el término 'medicalización' define un proceso que se extiende en la sociedad de nuestro tiempo, por el cual los médicos se ocupan y tratan problemas que atañen al bienestar humano, es decir problemas asociados a características intrínsecas de la vida, tales como: la sexualidad, la infelicidad, el deterioro biológico, el envejecimiento, la soledad y la muerte, entre otros. Así, problemas de la vida son tratados en términos de problemas médicos, es decir como enfermedades, síndromes o padecimientos.

El estudio de la medicalización es uno de los ejes centrales de los análisis de Foucault. En sus trabajos, analiza al saber médico como un discurso de poder, que operando en distintos dispositivos, forma parte del núcleo duro de las estrategias tendientes a la normalización de los cuerpos individuales y sociales. Pero además, inscribe a la medicina como participando en un fenómeno histórico más amplio: el proceso de medicalización de la sociedad (Foucault, 2003, 2000, 1996). Éste es entendido como proceso general a partir del cual en el siglo XVIII la medicina se convierte en específicamente social (Rosen, 1985; Castel, 1980), la medicina se torna una estrategia biopolítica, desplegando y expandiendo su dominio hacia cuestiones como el saneamiento del agua, las condiciones de vivienda, el régimen urbanístico, la sexualidad, la alimentación e higiene (Foucault, 1996). Estos ámbitos de incumbencia rebasan a los que antes convocaban a la medicina - centrados casi exclusivamente en la enfermedad y en la demanda del enfermo - dotándola de funciones de control y normalización de los cuerpos. El proceso de medicalización es pues esencial para la comprensión del modo en que los cuerpos - individuales y sociales - se vuelven objetos de saber, blancos del poder y campo de intervención de múltiples dispositivos.

Foucault abordó la medicalización partiendo de la hipótesis de que ningún problema es intrínsecamente médico o no. El dominio de la medicina no es único e inalterable, no está dado sino que

cada cultura define de una forma propia y particular el ámbito de los sufrimientos, de las anomalías, de las desviaciones, de las perturbaciones funcionales, de los trastornos de la conducta que corresponden a la medicina, suscitan su intervención y le exigen una práctica específicamente adaptada [...]. En último término la enfermedad es, en una época determinada y en una sociedad concreta, aquello que se encuentra práctica o teóricamente medicalizado. (Foucault, 1996, p.21)

Los aportes de Conrad resultan nodales para la comprensión del fenómeno de la medicalización. Conrad considera que la clave de este proceso está en la definición de un problema en términos médicos, utilizando un lenguaje médico para describirlo, adoptando un marco médico para entenderlo, y/o utilizando la intervención médica para tratarlo. El corolario de este fenómeno de medicalización es que "problemas no-médicos son definidos y tratados como problemas médicos, generalmente en términos de enfermedades o desórdenes" (Conrad, 1992, p.209). 
Otra de las contribuciones centrales de Conrad deriva de su análisis de las principales consecuencias de la medicalización para la sociedad. Dos son particularmente relevantes para el análisis que estamos realizando:

1 La expansión creciente de las áreas de incumbencia de la medicina, fomentada por una industria farmacéutica crecientemente poderosa y rentable;

2 El uso de diversas tecnologías por parte de la medicina para el tratamiento del comportamiento anormal. Estos mecanismos tecnológicos actúan de manera tal que contribuyen al sostenimiento del statu quo, con efectos que en ocasiones resultan irreversibles en quienes se ejercen.

Aunque estas contribuciones resultan relevantes para nuestros análisis, entendemos además junto a Conrad, que la consecuencia más trascendente de la medicalización es que retraduce las dificultades humanas en cuestiones individuales, omitiendo o relegando a un segundo plano la naturaleza social de los fenómenos. Y ello conduce a la obturación de otros niveles de intervención posibles. Según Conrad este enfoque en el organismo individual - que está en perfecta consonancia con la ética individualista de la cultura occidental - "deforma la realidad y permite el control social en nombre de la salud" (Conrad, 1982, p.154).

Expresando una de las posturas más radicales respecto de esta problemática, Thomas Szasz sostiene que la medicalización no es medicina, ni ciencia; es una estrategia semántica y social, que beneficia a algunas personas y daña a otras. El autor sostiene que si en el pasado las personas más injuriadas eran los pacientes con padecimientos mentales; hoy día la situación es más compleja, ya que cualquiera puede, en algún momento, ser alternativamente dañado por la medicalización (Szasz, 2007b).

Según Szasz, las sociedades occidentales actuales están guiadas y controladas por la ciencia, especialmente la ciencia médica. En este marco, la medicina y el Estado han formado un vínculo sólido, que el autor denomina "farmacracia" (Szasz, 2007a). Los análisis de Szasz corroboran y completan los efectuados casi treinta años antes por Foucault, quien había señalado en 1974 que en el futuro inmediato, la figura del médico adquiriría un rol cada vez más de mero intermediario entre el paciente y la industria farmacéutica (Foucault, 1996).

Los actuales estudios de Celia Iriart (2008) introducen un abordaje innovador y resignifican el rol de la industria farmacéutica a la luz de los procesos de la atención gerenciada en el sector salud. Iriart coloca al capital financiero como un nuevo actor en la reconfiguración de estos procesos. En este sentido la ordenación hegemónica del capital financiero que se produce en los '90s y las pujas distributivas que se generan, con el hasta entonces casi indiscutido actor central del sector salud, la industria farmacéutica, son determinante de las nuevas condiciones en que se produce la medicalización/ medicamentalización.

Iriart brinda elementos en el cual permite visualizar cómo

la puja distributiva no se ha resuelto entre el capital financiero y el complejo médico industrial, sino que ha adquirido nuevas formas que le dan a cada uno su parte, en desmedro del sector público, de los profesionales y de la salud de los colectivos sociales. (Iriart, 2008, p.1624)

Así la industria farmacéutica ha radicalizado el proceso medicalizador y medicamentalizador, produciendo nuevos desafíos analíticos del problema que aquí abordamos.

\section{Primera parte: el médico como intermediario. Los procesos de diagnóstico y tratamiento}

\section{Diagnóstico}

El TDA/H es un diagnóstico de expansión reciente en la Argentina. Según los profesionales entrevistados, habría dos fechas claves que marcan su auge, mientras algunos sostienen que el 
momento de crecimiento y consolidación ocurre durante los '90s, otros indican su incremento a partir del 2000, particularmente, en el período posterior a la crisis que experimentó la Argentina en el 2001.

Mencionamos previamente que uno de los principales debates en el campo del TDA/H es la existencia o no del diagnóstico "como tal". A lo largo de la investigación, hemos podido documentar una multiplicidad de posiciones respecto de la existencia y estatuto del diagnóstico.

Por un lado, y a pesar de admitir una importante imprecisión diagnóstica ligada al cuadro, una cierta confusión producto de diversos enmascaramientos diagnósticos y la alta frecuencia de la comorbilidad, la mayoría de los profesionales médicos entrevistados acuerdan en la existencia del cuadro clínico. Ellos afirman que es un diagnóstico antiguo y que las modificaciones se han dado sólo a nivel de la denominación, pero que su esencia se ha mantenido desde su primera descripción. Así mencionan que lo que hoy se conoce como TDA/H anteriormente era distinguido como "daño cerebral", "comportamiento amoral", "disfunción cerebral mínima", "hiperkinesia", "disritmia", "déficit de atención", "trastorno por déficit de atención".

Estas visiones mayoritarias, sin embargo, se contraponen con la perspectiva de otros entrevistados que rechazan la existencia misma del diagnóstico, atribuyendo a una conjugación de factores históricos, sociales y culturales la responsabilidad y la producción de la subjetividad del niño y por ende de su malestar. Algunos de estos entrevistados acuerdan que el TDA/H es una "moda", impuesta por los medios de comunicación o climas de época. Esta perspectiva enfatiza la subjetividad de cada niño y el contexto de producción familiar de sus síntomas o características de personalidad. Ciertos ideales epocales son mencionados como condicionantes en la producción de subjetividad del niño y su familia: "el rendimiento", "el éxito", "el consumo".

Por otro lado, algunos profesionales entrevistados hacen hincapié en los laboratorios como factores que contribuyen a la aparición del "sobrediagnóstico" y la "sobremedicación". Mencionan que el incremento de la incidencia del TDA/H es simultáneo con el momento en que se incorpora en mayor medida la práctica de medicar a los niños.

Hubo distintas respuestas según la especialidad médica de los entrevistados en lo atinente a la etiología del cuadro, la configuración del diagnóstico del TDA/H, y la terapéutica delineada para el mismo. También se observaron diferencias en torno a la magnitud de la consulta en los subsectores, público y privado, de atención a la salud. Sin embargo, una consideración importante y consensuada de la construcción diagnóstica son las enunciaciones acerca de que la enfermedad no se cura, sino que sólo puede aspirarse a mantenerla controlada (con o sin medicación mediante). Esta apreciación de la enfermedad conlleva a que el tratamiento (en sentido amplio, como necesidad de estar en contacto con el ámbito médico) sea "de por vida".

Ahora bien, acerca de la etiología del síndrome no parece haber certezas ni acuerdos entre los entrevistados. En líneas generales se habla de multicausalidad para explicarlo. Sin embargo, los especialistas en neurología infantil señalan enfáticamente causas ligadas a compromisos orgánicos y /o predisposiciones genético-hereditarias.

Aquellos profesionales que trabajan en el sector público estatal de salud, refieren atender casos que revisten, a su atender, mayor complejidad que el TDA/H. Plantean la posibilidad de sub-diagnóstico en poblaciones con menores recursos socioeconómicos, lo cual asocian a la presentación de otros problemas sociales complejos en la infancia, la adolescencia o en la edad adulta. Asimismo, señalaron que la mayoría de los niños con diagnóstico de TDA/H eran atendidos en efectores privados de salud.

En la construcción diagnóstica, y a diferencia de otros padecimientos, el fármaco ocupa un lugar central. Los entrevistados, principalmente los profesionales de la neurología infantil, señalan que el fármaco puede ser utilizado como una herramienta para la constitución misma del diagnóstico, es decir el consumo del fármaco anticipa el diagnóstico del TDA/H. La confirmación diagnóstica a partir del consumo del fármaco, se formula bajo la premisa "si el niño funciona con el medicamento, estamos ante un caso que podemos diagnosticar como TDA $/ \mathrm{H}^{\prime}$. Tal como lo referían en ocasiones otros participantes del estudio, "este procedimiento estaría alterado el circuito esperable de detección, diagnóstico y tratamiento, dado que el fármaco se constituye en el eje del diagnóstico y no sólo en una posible terapéutica", con los riesgos ligados a sobremedicación que esto conlleva. 
A lo largo de las entrevistas, la escuela se revela como la principal fuente de derivación al médico. La derivación puede tener dos direcciones: hacia el pediatra de cabecera o hacia un especialista, habitualmente un neurólogo infantil, y por lo general su nombre o la institución de pertenencia viene sugerida por la propia escuela.

A partir de esta primera consulta se inicia un circuito de re-derivaciones que varía según los profesionales intervinientes. Más que por razones de índole clínica, el circuito de diagnóstico y tratamiento queda definido por los profesionales y sus posturas terapéuticas, evidenciándose aquí una división entre los que son a) proclives a la medicación o son b) proclives a terapias no medicamentosas, muchos de los cuales se autodefinen como "anti-medicación". En función de esta línea se juega cada uno de los circuitos que el niño va a ir atravesando.

Como vemos, frente a un niño diagnosticado con TDA/H, los distintos actores médicos vuelven a poner de manifiesto ante el tratamiento la división ya constatada en el relevamiento de la temática diagnóstica: medicar o no. Es en función de esta gran divisoria de aguas que se interconsultan los distintos profesionales médicos con el fin de precisar el diagnóstico y asegurarse el camino terapéutico adecuado. Más que por la necesidad y requerimiento del cuadro clínico que puede plantear un niño, el circuito terapéutico parece definirse por las posturas personales de los profesionales en juego ante la medicación.

\section{Tratamiento}

Una vez que el niño es diagnosticado, puede iniciar un recorrido que se disputa entre los dos grupos ya señalados; sin embargo la construcción propia del diagnóstico de TDA/H implica una asociación terapéutica con el fármaco.

Así, a pesar de que todos los entrevistados médicos señalan un cierto consenso o manifiestan las ventajas de tratamiento integral - es decir, no centrado exclusivamente en el psicofármaco -, en un grupo de profesionales, particularmente integrados por neurólogos infantiles, el fármaco emerge como principal regulador del tratamiento y el resto de las terapéuticas se enuncian como subsidiarias de ésta.

Un aspecto que nos ha llamado la atención en los circuitos de derivación y tratamiento, es que el psiquiatra infanto-juvenil no se encontró asociado a esta problemática en su etapa diagnóstica y de inicio del tratamiento. Su intervención está relacionada con interconsultas a partir de fracasos en los tratamientos farmacológicos, donde se piensa una comorbilidad asociada a una psicopatología de base más grave.

Los pediatras constituyen un colectivo de profesionales que siguen manteniendo una mirada integral tanto a la hora de diagnosticar como de indicar una terapéutica, y en general, manifiestan buscar alternativas a la prescripción de medicamentos. Sin embargo, la reconstrucción de los circuitos de tratamiento pone de manifiesto que la pediatría es una especialidad central en los procesos y decisiones de derivación hacia otras especialidades y por lo tanto un engranaje clave en la direccionalidad que adoptará el camino terapéutico. De la derivación depende la disciplina profesional que se consultará para abordar el problema (neurología, psicología, psicopedagogía, etc.). Al mismo tiempo, de esta derivación se desprende en gran medida si el abordaje estará basado o no en la medicación, pues como vimos, el circuito terapéutico depende en gran medida de las posturas personales en torno al síndrome y al abordaje que cada profesional adopta.

En relación a prescripción del fármaco, el nombre de fantasía más mencionado por los entrevistados fue la Ritalina ${ }^{\circledR}$ (metilfenidato), droga de venta restringida y controlada en la Argentina por la ANMAT. En relación al genérico atomoxetina, el nombre comercial más aludido fue el Recit ${ }^{\circledR}$.

Si bien en términos generales cada médico es proclive a la selección de una de las drogas utilizadas, hemos detectado que hay profesionales que distinguen el tipo de droga a la hora de medicar: atomoxetina para aquellos casos en los cuales hay predominio de impulsividad y el metilfenidato para aquellos diagnósticos en los cuales la desatención es la principal característica.

La administración de la medicación sigue los movimientos del ciclo escolar: de lunes a viernes (con descanso durante el fin de semana) y de marzo a diciembre. Todos los médicos prescriptores de estos psicofármacos refieren utilizar este diagrama de consumo. 
Respecto de los tiempos del tratamiento medicamentoso se ha encontrado mucha diversidad e imprecisión: hay quienes sostienen que dos o tres años de tratamiento medicamentoso es suficiente; otros afirman que conviene mantenerlo durante toda la escolaridad; otros, en cambio sostienen la necesidad de evaluar cada año, para decidir si se interrumpe la medicación en función de los cambios que se hubieran provocado.

Se registra una naturalización entre la administración de la medicación y el ciclo escolar. Al mismo tiempo, los distintos actores mencionan la presión de los padres y del colegio a la hora de evaluar la indicación psicofarmacológica.

En las narraciones de los profesionales el consumo de medicación para el TDA/H se encuentra asociado a los niveles socioeconómicos de pertenencia de los niños. Si bien hemos encontrado una difundida naturalización del consumo de psicofármacos para niños con este diagnóstico, dicha naturalización parece ser más relevante en los sectores sociales medios o altos. Se menciona que en escuelas de mayores recursos socio-económicos el consumo de psicofármacos para el TDA/H se encuentra ampliamente naturalizado y extendido. Los entrevistados infieren que esto estaría asociado a que estos niños están más expuestos que sus pares de bajos recursos a expectativas de exigencia y rendimiento (social y académico) y el consumo de psicofármacos estaría directamente inscripto en el cumplimiento de tales expectativas. Refiere un entrevistado que "el fármaco se convierte en una alternativa frecuentemente solicitada por los padres; en general, se puede rastrear que son adultos en quienes es posible indagar ideales similares y en quien también recaen las mismas exigencias de éxito".

En este mismo sentido varios profesionales entrevistados señalaron que la medicación resulta una herramienta vinculada a la "comodidad", y está en "consonancia con la exigencia de rendimiento y eficacia". Esto podría estar asociado a un proceso de sobrediagnóstico dado que no es un argumento estrictamente clínico el que se está tomando para juzgar la necesidad de la medicación en el niño, sino que priman los factores institucionales, familiares y sociales. Al mismo tiempo, los profesionales entrevistados explican la extensión de la medicación en estos sectores de mayores recursos por la cobertura en salud y la posibilidad de acceder económicamente a la medicación.

Por el contrario, en sectores más vulnerables y marginales tanto la desatención de un niño o su hiperactividad son referenciadas, o bien como "problemas de conducta", o bien como "característica de personalidad del niño". Según un entrevistado, a estos niños no se le atribuyen "expectativas o posibilidades para aprender, sino que sólo se espera que no provoquen disturbios o desórdenes en el aula y puedan sostenerse en el sistema escolar primario". Al mismo tiempo no existen, tanto a nivel nacional como de las provincias en las cuales se realizó el trabajo de campo, programas estatales de distribución de medicamentos en los cuales se incluya el metilfenidato y/o la atomoxetina.

Un hallazgo resultante de la investigación fue la aparición de casos de administración de neurolépticos y antidepresivos en niños con presunto diagnóstico de TDA/H. Esto introduce cierto cuestionamiento dado que ambos medicamentos no fueron autorizadas por Food \& Drugs Administration (FDA) para la prescripción en diagnóstico de TDA/H, y sólo algunos de ellos están autorizados para ser prescriptos niños mayores de cinco años con otros diagnósticos. Sólo uno de los informantes claves se refirió a la escasez de estudios científicos rigurosos y extendidos en el tiempo en la población infantil en relación a estos fármacos, hecho que pone de manifiesto problemas éticos y bioéticos en relación a la prescripción y política de medicación en nuestro país.

\section{Segunda parte: el reposicionamiento de la industria farmacéutica}

\section{Marketing e industria farmacéutica}

Durante el desarrollo de la investigación nos interesó indagar acerca del modo en que la industria farmacéutica en la Argentina consolida estrategias de marketing en el mercado de los psicofármacos para niños, a fin de analizar la articulación entre estas estrategias, el diagnóstico y tratamiento del TDA/H y la consolidación de los procesos de medicalización/medicamentalización. 
${ }^{9}$ Información suministrada en entrevista por el Departamento de Psicotrópicos y Estupefaciente de la ANMAT.

\footnotetext{
${ }^{10}$ Debemos destacar que el acceso a las entrevistas a Personal Jerárquico de los Laboratorios, Coordinadores de Área y Agentes de Propaganda Médica fue dificultosa. Así mismo, en las entrevistas que accedimos no se nos permitió realizar grabaciones.
}

El TDA/H en la población infantil es un mercado en expansión, tal como lo muestran, por un lado, las cifras de importación de metilfenidato proporcionadas por el ANMAT que señalan un significativo incremento en la importación de esta droga en la Argentina, de $47.91 \mathrm{~kg}$. en el 2007 a $81.75 \mathrm{~kg}$. en el $2008^{9}$; y por otro, las estrategias de marketing que la industria farmacéutica implementa.

A lo largo de la investigación observamos que el marketing farmacéutico se dirige por un lado hacia la comunidad médica, a través de las ya conocidas estrategias de incentivos y/o controles, y también a través mecanismos de difusión de información a través de los líderes de opinión o "speakers". Pero también, detectamos que la industria farmacéutica pone especial énfasis en estrategias de marketing dirigidas a usuarios y/o sus organizaciones de familiares y a la comunidad educativa. Si bien estas acciones están prohibidas por la legislación nacional, se crean intersticios que posibilitan llevarlas a cabo.

Hemos sistematizado los procedimientos de marketing que la industria farmacéutica implementa en relación a las dos drogas para el tratamiento del $\mathrm{TDA} / \mathrm{H}^{10}$. Así, teniendo en cuenta las tácticas y los sujetos destinatarios pudimos distinguir diversas y novedosas estrategias.

\section{Estrategias dirigidas a los médicos}

Esta estrategia está relacionada con el seguimiento minucioso del médico. Este actor privilegiado en la cadena de transmisión para el consumo del fármaco está cada vez más objetivado por el propio proceso de medicalización - medicamentalización (Pavlovsky, 2006). Los laboratorios pueden hoy tener un seguimiento minucioso sobre qué y cuándo recetan los médicos a través de información construida por empresas de mercadotecnia (Jara, 2007). En la Argentina pudimos rastrear que dos son las principales. Sobre una de ellas, Lakoff (2003) realizó un interesante seguimiento acerca del modo en que este tipo de empresas operan para identificar a los médicos, las drogas y cantidades prescriptas a través de la copia de recetas microfilmadas tomadas en las grandes cadenas farmacéuticas de la ciudad de Buenos Aires. Durante la investigación hemos podido acceder a información que estas empresas venden a los laboratorios, particularmente relacionadas con fármacos para el TDA/H. Esta información se elabora mensualmente y revela las cifras de unidades vendidas (cajas vendidas) vinculadas a la totalidad de fármacos utilizados para este síndrome - tanto por el laboratorio que compra el resumen, como por los otros laboratorios competidores en el mercado. Según información de representantes de los propios laboratorios, estas cifras son las de mayor exactitud de venta, constituyéndose en la información más limpia que se puede rastrear en materia de estudios de mercado.

A lo largo del trabajo de campo se observó que la estrategia dirigida a los médicos ha cambiado sustancialmente respecto de lo conocido hace tiempo atrás, ya no es necesario visitar día a día al médico para motivarlo y/o vigilarlo, otros mecanismos de control se ponen en juego. Si bien los APM casi no visitan a los pediatras por temas relacionados con el campo de la salud mental, estos se encuentran muy informados acerca del TDA/H a través de publicaciones de los laboratorios y las denominadas "guías de consenso".

En relación a los distintos especialistas médicos podemos decir que cuando se trata de psicofármacos para niños en general, los psiquiatras infanto juveniles y los neurólogos infantiles son las especialidades a las cuales se dirigen propagandas y/o privilegios por parte de los laboratorios. En el caso específico del TDA/H, según la información recabada, son los neurólogos infantiles los actores más relevantes. 


\section{Lideres de opinión}

Los líderes de opinión son un engranaje clave en la actual estrategia de marketing de la industria farmacéutica. Según información vertida por los APM, son ellos mismos quienes evalúan a los médicos según su rol con los semejantes, luego son los propios laboratorios quienes los "moldean" a fin de elevarlos a la categoría de expertos, o, tal como lo definió un informante clave, en "sabios locales". Son también los encargados de difundir los últimos hallazgos en relación a enfermedades y/o síndromes y las posibles terapéuticas en congresos, conferencias científicas, publicaciones y/o a través de las guías de consenso. Relatan Moynihan y Cassels (2006) que las alianzas con los líderes de opinión son tan importantes que algunas empresas de marketing calculan el rendimiento de la inversión que una campaña farmacéutica puede cosechar de esta clase de presentaciones.

De acuerdo a las fuentes consultadas y las entrevistas realizadas a informantes claves de la industria farmacéutica, se destacó la estrategia de los líderes de opinión, o "speakers", para la difusión de la información sobre la medicación del TDA/H a otros miembros de la comunidad médica. Según lo relevado, estos líderes de opinión son médicos psiquiatras infanto juveniles y neurólogos infantiles destacados pertenecientes a las instituciones públicas y/o privadas de mayor "prestigio" asistencial de la ciudad de Buenos Aires. Un entrevistado perteneciente a la industria farmacéutica expresa:

“cada laboratorio tiene sus líderes de opinión que son los que sostienen científicamente (con teorías) la tendencia del laboratorio en materia de fármacos. Cada uno se lleva un cheque...o equivalentes. Hay laboratorios que tienen políticas de captación de psiquiatras y otras que premian por lo que los psiquiatras ya recetan. Nosotros ya sabemos que hay médicos que con tal de que les pagues dicen cualquier cosa.... En el medio ya se los conoce.... y a esos se apuesta".

\section{Estrategia directa hacia los usuarios y/o sus organizaciones}

En la Argentina la alianza entre la industria farmacéutica y ciertas asociaciones de pacientes/ padres y/o familiares es aún muy incipiente y no tiene el desarrollo que ha adquirido en otros países. Sin embargo, se observa la tendencia de algunos laboratorios a organizar grupos de dos, tres o más familias con el objetivo de brindar información sobre el TDA/H. A estas reuniones se las suelen denominar "mesas de ayuda", muchas veces promocionadas a través de las escuelas y generalmente realizadas en algún tipo de ámbito médico no asistencial.

Otra estrategia detectada dirigida a la familia fue la enunciada por los entrevistados como "los incentivos". Consiste en la captación del interés hacia una droga en particular a través de la provisión de incentivos durante un tiempo. Estos incentivos están asociados a la entrega de cajas compensatorias si se selecciona un determinado fármaco de cierto laboratorio.

\section{Estrategias dirigidas a la comunidad educativa}

A lo largo del trabajo de campo pudimos rastrear el intento de la industria farmacéutica por acercarse a los ámbitos educativos, particularmente a docentes y gabinetes psicopedagógicos. La escuela es un actor privilegiado, como ya se expresó, para iniciar el circuito de derivación y tratamiento del TDA/H.

La investigación permitió observadar tres modalidades estratégicas que los laboratorios implementan hacia la comunidad educativa.

La primera podría definirse como aquella en la cual los empleados o agentes de los laboratorios, APM, que junto $a$, o a través de, especialistas médicos concurren a las escuelas y/o realizan charlas informativas a los gabinetes psicopedagógicos. Al igual que en las estrategias con las familias, a estos encuentros se los denominan "mesas de ayuda".

La segunda estrategia identificada fue la elaboración de información destinada a maestros. Esto se verificó en la edición de cuadernillos destinados a profesionales del ámbito educativo, con consejos para docentes e información acerca de los medicamentos a utilizar para el TDA/H. 
Por último también observamos la difusión de las drogas disponibles en el mercado en revistas pedagógicas. Hemos relevado números completos dedicados al TDA/H en revistas especializadas para docentes y psicopedagogos. En ellas, los autores son generalmente líderes de opinión de laboratorios vinculados a la producción de drogas utilizadas para este síndrome.

\section{Conclusiones}

En este artículo analizamos el avance de los procesos de medicalización/medicamentalización en la infancia a partir de un analizador: el Trastorno de Déficit de Atención con o sin Hiperactividad (TDA/H).

La medicalización como forma de intervención política se presenta como continua e indefinida y busca abarcar todo lo concerniente a la vida social en el mayor rango de edad posible, de tipo de población, y con la mayor duración posible en el tiempo. A su vez, la medicina ya no se ocupa de combatir la enfermedad, sino de hacer vivir de determinada manera. Su campo de acción no es sólo el de la enfermedad, sino el de la vida como un todo. La medicalización de la vida cotidiana resulta un proceso de larga data, que en la actualidad toma una forma específica: la medicamentalización.

El consumo de psicotrópicos en niños diagnosticados con TDA/H es un modelo de cómo ciertas conductas y/o situaciones que antes no eran medicalizadas hoy son parte de los circuitos de tratamiento médico, circuitos que tienen principal énfasis en el consumo de fármacos como terapéutica.

La Argentina no cuentan con estadísticas de diagnostico de TDA/H, sin embargo a lo largo de la investigación hemos registrado, según datos proporcionados por ANMAT, un significativo incremento en la importación del metilfenidato (47.91 kg. en el 2007, 81.75 kg. en el 2008). Dichas cifras, sumado al discurso de los entrevistados del campo médico y a las estrategias de marketing que la industria farmacéutica desarrolla para este síndrome, nos ubica en un escenario en el cual podríamos concluir que en Argentina hay auge y consolidación del diagnóstico de TDA/H en la población infantil.

En relación al debate sobre la existencia o no del TDA/H como diagnóstico hemos documentado que la mayoría de los profesionales médicos de las tres especialidades abordadas en esta investigación, acordaron en la existencia del TDA/H como entidad clínica tal lo describe el DSM IV. Sin embargo la mayoría reconoce una dificultad para precisar el diagnóstico y distinguirlo de otros cuadros. En menor medida, algunos profesionales afirman que el TDA/H existe como producto de la moda o como invención de la industria farmacéutica. Asimismo, se observó una diferenciación diagnóstica según el sector social de pertenencia de los niños, una preponderancia del diagnóstico en sectores medios altos y una menor participación de los sectores bajos. En este último, la fenomenología del TDA/H en general no es interpretada en términos diagnósticos, sino como "problemas de conducta" o como "características de la personalidad del niño".

Si bien se registraron distintas posiciones según especialidad médica y subsector de pertenencia de los entrevistados respecto del tratamiento, el fármaco emerge como la opción terapéutica mayoritaria. Según las narraciones profesionales, entre las distintas disciplinas médicas la neurología infantil es la que más tiende a la prescripción de fármacos.

El circuito de tratamiento donde se inscribe la decisión de medicar, está dado por la escuela que la propone, la familia que la solicita y el médico que la receta.

Por otro lado, se ha identificado que la industria farmacéutica viene implementando nuevas estrategias de marketing y difusión (líderes de opinión, mesas de ayuda, gacetillas, etc.). Estas estrategias, que se suman a las ya conocidas destinadas a la comunidad médica, se dirigen a actores no médicos, particularmente maestros y asociaciones de padres. De este modo, la industria farmacéutica y sus productos de mercado encuentran nuevos modos de penetrar en la comunidad educativa y en las familias, áreas principales donde se desarrolla la vida del niño.

El análisis del TDA/H permite transparentar cómo ciertas manifestaciones de vida de los niños son pensadas como enfermedades, síndromes o padecimientos e ilustrar el modo en que la industria farmacéutica reconstruye estas configuraciones en términos de ganancia. 


\section{Colaboradores}

Los autores trabajaron juntos en todas las etapas de la producción del manuscrito.

\section{Referências}

BENASAYAG, L. (Comp.). ADDH: niños con déficit de atención e hiperactividad. ¿Una patología de mercado? Una mirada alternativa con enfoque multidisciplinario. Argentina: Noveduc, 2007.

BRIO, M.C. Controversias y cuestiones éticas con el uso de psicofármacos en niños y adolescentes. In: CONGRESSO DE APSA, 13., 2007, Argentina. Ponencia... Mar del Plata, 2007.

CASTEL, R. La sociedad psiquiátrica avanzada: el modelo norteamericano. Barcelona: Anagrama, 1980.

CONRAD, P. Medicalization and social control. Annu. Rev. Sociol., n.18, p.209-232, 1992.

Sobre la medicalización de la anormalidad y el control social. In: INGLEBY, D. (Ed.). Psiquiatría crítica: la política de la salud mental. Barcelona: Crítica-Grijalbo,1982. p.129-54.

DSM-IV. Breviario: criterios diagnósticos. Barcelona: Masson, 1995.

FARAONE, S. Medicalización de la infancia: una mirada desde la complejidad de los actores y de las políticas. 2008. Disponible en: <http://www.topia.com.ar/autores/silviafaraone >. Acceso en: 22 fev. 2010.

FOUCAULT, M. Seguridad, territorio, población. Argentina: Fondo de Cultura Económica, 2006

El poder psiquiátrico. Argentina: Fondo de Cultura Económica, 2005.

Historia de la sexualidad. Argentina: Siglo XXI, 2003. T.1.

Los anormales. Argentina: Fondo de Cultura Económica, 2001.

Defender la aociedad. Argentina: Fondo de Cultura Económica, 2000.

La vida de los hombres infames. Buenos Aires: Altamira, 1996.

ILLICH, I. Némesis médica: la expropiación de la salud. Barcelona: Barral Editores, 1975.

IRIART, C. Capital financiero versus complejo médico-industrial: los desafíos de las agencias regulatorias. Cienc. Saude Colet., v.13, n.5, p.1619-26, 2008.

JANIN, B. El ADHD y los diagnósticos en la infancia: la complejidad de las determinaciones". Rev. Cuestiones Infanc., n.11, p.15-35, 2007. Disponible en: <http:// www.forumadd.com.ar/documentos/t11.htm >. Acceso en: 22 fev. 2008.

JARA, M. Traficantes de salud: cómo nos venden medicamentos peligrosos y juegan con la enfermedad. Barcelona: Icaria-Antrazyt, 2007.

LAKOFF, A. La ansiedad de la globalización: venta de antidepresivos y crisis económica en la Argentina. Cuad. Antropol. Soc., v.18, p.35-66, 2003.

MICHANIE, C. Trastorno por déficit de atención e hiperactividad. En Terremotos y soñadores. TDAH J., v.1, n.1, p.6-9, 2000.

MOYNIHAN, R.; CASSELS, A. Medicamentos que nos enferman e industria farmacéutica que nos convierten en pacientes. Barcelona: Edición Terapia Verde, 2006 
PAVLOVSKY, F. La tentación: vicisitudes de un psiquiatra. Rev. Topía, n.47, 2009.

Disponible en: <http://www.topia.com.ar/articulos/la-tentaci\% C3\%B3n-visicitudes-deun-psiquiatra >. Acceso en: 22 fev. 2010.

ROSEN, G. De la policía médica a la medicina social: ensayos sobre la historia de la atención a la salud. México: Siglo XXI, 1985.

SCANDAR, R. Inquietos, distraídos, ¿diferentes? Guía para padres y docentes de niños con TDA/H. Buenos Aires: Editorial EDIBA, 2007.

SZASZ, T. Medicalizing quackery. Freeman, n.57, p.22-3, 2007a. Disponible en: < http:// www.szasz.com/freeman20.html>. Acceso el: 22 feb. 2010.

The medicalization of everyday life. Freeman, n.57, p.18-9, 2007b. Disponible en: <http://www.szasz.com/freeman23.html>. Acceso el: 22 fev. 2008.

VASEN, J. Fantasmas y pastillas: intervenciones psicoanalíticas y psicofarmacológicas con niños. Buenos Aires: Letra Viva, 2005.

FARAONE, S. et al. Discurso médico e estratégias de marketing da indústria farmacêutica nos processos de medicalização da infância na Argentina. Interface - Comunic., Saude, Educ., v.14, n.34, p.485-97, jul./set. 2010.

Neste artigo exploram-se processos de medicalização e medicamentalização na infância, utilizando como analisador o Transtorno de Déficit de Atenção com ou sem Hiperatividade (TDA/H). Apresentam-se os resultados de um estudo qualitativo realizado por uma equipe interdisciplinar durante 2008, em quatro jurisdições argentinas: Região Metropolitana de Buenos Aires, Corrientes, Salta e Tierra del Fuego. Exploram-se os discursos do campo médico - pediatras, psiquiatras infanto-juvenis e neurologistas infantis - em torno da construção diagnóstica do TDH/A e da sua abordagem terapêutica, nos sistemas público e privado. De modo complementar, indaga-se acerca dos mecanismos de marketing da indústria farmacêutica. Por meio da construção de diagnósticoTDA/H, é possível observar como determinadas condutas e/ou situações, que antes não eram medicalizadas, hoje são parte do tratamento médico, cuja principal ênfase encontra-se na prescrição de fármacos como terapêutica.

Palavras-chave: Infância. Medicalização/medicamentalização. Psicofármacos.

Recebido em 31/08/2009. Aprovado em 09/02/2010. 
